\title{
Roxithromycin as Anti-Inflammatory Drug Improves Clinical Outcomes in Adult Patients with Bronchiectasis: A Double- Blinded Randomized, Placebo-Controlled Pilot Clinical Trial
}

\author{
Siwasak Juthong, M.D., Sarayuth Eiamsa-ard, M.D. \\ Division of Respiratory and Respiratory Critical Care Medicine, Department of Internal Medicine, Faculty of Medicine, \\ Prince of Songkla University, Hat Yai, Songkhla 90110, Thailand.
}

Received 1 February 2019 • Revised 23 April 2019 • Accepted 24 April 2019 • Published online 24 June 2019

\section{Abstract:}

Objective: Macrolides are anti-inflammatory agents that have been reported to improve symptoms in bronchiectasis. The objectives of this study were roxithromycin 300 milligrams $(\mathrm{mg})$ once daily for 8 weeks in patients with bronchiectasis improves the symptom scores as the primary outcome, and the pulmonary function tests and quality of life as the secondary outcomes.

Material and Methods: This was a randomized double-blinded placebo-controlled trial of roxithromycin or placebo once daily for 8 weeks in patients with bronchiectasis. Symptom scores, pulmonary function tests, St. George’s Respiratory Questionnaire (SGRQ), and sputum culture were done at each visit.

Results: Twenty-eight bronchiectasis patients with a mean age of 56 years participated in this study. Fourteen patients were randomized to receive either roxithromycin $300 \mathrm{mg}$ or placebo once daily. Roxithromycin was shown to improve symptom scores (mean difference of symptom scores $=-1.66$, p-value=0.005) compared with placebo (mean difference of symptom scores $=-0.06$, p-value=0.94). Quality of life assessed by the $S G R Q$, significantly improved in the roxithromycin group ( $p-v a l u e=0.012$ ) but not in the placebo group $(p-v a l u e=0.84)$. Pulmonary function tests (forced expiratory volume in one second, forced vital capacity, and diffusing lung capacity of the lung for carbon monoxide) did not improve in either group. None of the patients in the roxithromycin group reported any adverse effects.

Conclusion: Roxithromycin once daily showed benefits in clinical outcomes such as symptoms and quality of life. Larger studies of the effects of roxithromycin in bronchiectasis with longer follow-up should be done.

Keywords: anti-inflammatory, bronchiectasis, macrolide, roxithromycin, treatment

Contact: Siwasak Juthong, M.D.

Division of Respiratory and Respiratory Critical Care Medicine,

Department of Internal Medicine, Faculty of Medicine,

Prince of Songkla University, Hat Yai, Songkhla 90110, Thailand

Email: jsiwasak@medicine.psu.ac.th
J Health Sci Med Res 2019;37(3):229-236 doi: 10.31584/jhsmr.201956 www.jhsmr.org 


\section{Introduction}

Bronchiectasis is a chronic inflammatory airway disease characterized by progressive symptoms of productive cough, shortness of breath, occasional hemoptysis, and repeated infection that requires antibiotics. ${ }^{1}$ The illness is related to retained inflammatory secretion containing microbes that cause obstruction and damage to the airway and recurrent infection. ${ }^{2}$ The Cole's vicious cycle evolution pathogenesis causes bronchiectasis patients to worsen gradually with progressive deterioration of overall symptoms with a poor quality of life. ${ }^{3}$ Many inflammatory cells and proinflammatory cytokines play roles in bronchiectasis. ${ }^{4}$ Long-term macrolide therapy has many beneficial effects on the clinical outcomes in chronic inflammatory airway diseases. ${ }^{5}$ Long-term macrolide antibiotics (e.g., erythromycin $^{6}$ and azithromycin as anti-inflammatory agents ${ }^{7,8}$ ) in bronchiectasis patients with three or more exacerbations per year significantly decreased pulmonary exacerbations.

Roxithromycin is an effective macrolide with potent antiinflammatory effects that decreases the release of chemotactic mediators which prolong the half-life of roxithromycin, enhances the stability in gastric juice, has safe long-term side effects, and is low in price. ${ }^{9}$ Roxithromycin improved the clinical symptoms of bronchiectasis patients in one small study. ${ }^{8}$ However, it was not a randomized controlled trial of roxithromycin therapy in adult bronchiectasis patients and did not mention quality of life. In this pilot study we hypothesized that once daily roxithromycin 300 milligrams $(\mathrm{mg})$ for 8 weeks in patients with bronchiectasisimproves the symptom scores as the primary outcome. Pulmonary function tests and quality of life assessments were the secondary outcomes.

\section{Study design}

This study was a double-blind randomized controlled trial. Block-of-four randomization was used and allocation concealment was by sealed packages of the drug and placebo.

\section{Patients}

The study was conducted between June 1, 2010 and November 30, 2010 in the outpatient clinic at Songklanagarind Hospital in southern Thailand. The inclusion criteria were symptomatic non-cystic bronchiectasis patients diagnosed by high resolution computed tomography. The patients were more than 18 years old and had a history of infectious exacerbation of bronchiectasis more than one time in the previous 12 months. The exclusion criteria included documented active pulmonary infection within the previous 3 months, active malignancy, and known allergy to macrolide antibiotics. This study was approved by the hospital ethics committee.

\section{Materiall and Methods}

Roxithromycin $300 \mathrm{mg}$ tablets were manufactured by Pharmasant Laboratories Co., LTD., Thailand. Placebo tablets were manufactured and packed by a pharmacist in the Department of Pharmaceutical Manufacturing, Faculty of Pharmaceutical Sciences, Prince of Songhkla University, Thailand.

\section{Study protocol}

All participants signed the informed consent forms. They were then randomized to receive either roxithromycin $300 \mathrm{mg}$ once daily or the placebo for 8 weeks. All patient baseline characteristics including age, smoking status, current and past chest symptoms, possible etiology of bronchiectasis, and concurrent respiratory medications were recorded. Bronchiectasis was assessed and scored according to the symptoms ${ }^{9}$ shown in Table 1 supplement. The score range was from 0 to 12. Higher scores indicated more symptoms. Data recorded at the first visit and at the 
Table supplement 1 Bronchiectasis symptom scores*

\begin{tabular}{lllll}
\hline \multirow{2}{*}{ Score } & \multicolumn{3}{c}{ Clinical symptoms and signs } \\
\cline { 2 - 4 } & Cough & Sputum & Wheeze & Dyspnea \\
\hline 3 & Persistent & Purulent $\geq 10$ times/day & Diffuse, severe & At rest \\
2 & Frequent & Purulent $<10$ times/day & Diffuse, Mild & On mild exertion \\
1 & Occasional & Serous & Localized, Mild & On moderate exertion \\
0 & None & None & None & None \\
\hline
\end{tabular}

*Modified from Nakamura H, Fujushima S, Inoue T, Ohkubo Y, Soejima K, Waki Y, et al. Clinical and immunoregulatory effects of roxithromycin therapy for chronic respiratory infection. Eur Respir J 1999;13;1371-9.

8 week visit at the end of the study protocol included the St. George Respiratory Questionnaire (SGRQ), pulmonary function tests [i.e. forced expiratory volume in one second $\left(F E V_{1}\right)$, forced vital capacity (FVC), and diffusing lung capacity of the lung for carbon monoxide (DLCO)]. Sputum cultures for aerobic bacteria were performed at the first visit and at the end of the study protocol. Surveillance of potential adverse drug effects was recorded. All participants were informed of pulmonary rehabilitation (e.g., breathing exercise and postural drainage) and they continued to use prior medications. The primary outcome was the improvement of symptom scores. The secondary outcomes were pulmonary function tests, quality of life, safety, tolerability, and emerged drug-resistance strains.

\section{Statistical analysis}

Demographic data are reported as mean \pm standard deviation (S.D.) for numerical and normally distributed data and as median with minimal and maximal values if skewness of data was found. Primary end points (symptom scores) and secondary end points (lung function tests, safety, tolerability, and emerged drug-resistant strains) are presented as mean \pm S.D. A comparison of normally distributed data of basal characteristics between both groups was performed by parametric statistical t-test and Wilcox log rank test or Mann-Whitney $U$ test in case of non-parametric data. $P$-values $<0.05$ were considered as statistically significant. Statistical Package for the Social Sciences version 11.5 was used for the statistical evaluation.

\section{Results}

Thirty-three patients were enrolled in the study but 5 patients were excluded. One patient refused to give informed consent, 1 patient participated in another trial, 1 patient had active malignancy, and 2 patients had taken roxithromycin within the previous 3 months. Twenty-eight non-cystic bronchiectasis patients were randomized to either the roxithromycin or placebo group (Figure 1). The patient demographics and clinical details are shown in Table 1. Fourteen non-cystic bronchiectasis patients received roxithromycin $300 \mathrm{mg}$ once daily and the other 14 patients received an identical placebo. In the end 12 patients were analyzed because 2 patients were lost to follow-up due to local flooding.

Table 1 shows the baseline characteristics and concomitant use of respiratory medications. There were no statistical differences in the baseline characteristics between the roxithromycin and placebo groups. The most common cause of bronchiectasis was post-tuberculosis. Concurrent respiratory medications were inhaled cortico- 
steroid, long acting theophylline, and a mucolytic. The mean symptom scores at the first visit in the roxithromycin and placebo groups were $5.33 \pm 1.72$ and $5.35 \pm 1.98$ points, respectively ( $p-$ value $=0.138)$. The mean symptom scores at week 8 were $3.67 \pm 1.82$ points in the roxithromycin group and $5.29 \pm 2.98$ points in the placebo group. The mean difference in the symptom scores between the first week and week 8 were $-1.66 \pm 1.23$ points in the roxithromycin group and $-0.06 \pm 2.34$ in the placebo group $(p-$ value $=$ 0.027). A comparison of the improvement of symptom scores in each group with the baseline was significantly different only in the roxithromycin group ( $p$-value $=0.005$ compared with the placebo group with a $p$-value=0.94) (Table 2). There were no statistically significant differences in the baseline pulmonary function tests between the roxithromycin and placebo groups (Table 3). In the roxithromycin and placebo groups the means of the $\mathrm{FEV}_{1}$ were $1.53 \pm 0.62$ liters and $1.31 \pm 0.44$ liters $(p-$ value $=0.613$ ), the means of the FVC were $2.27 \pm 0.79$ liters and $1.98 \pm 0.55$ liters $(p-v a l u e=0.282)$, and the mean percentages of the diffusing capacity of the lung for carbon monoxide (\%DLCO) were $84.0 \pm 15.6 \%$ and $83.9 \pm 30.2 \%$ (p-value=0.843), respectively. No statistically significant improvements in lung functions were noted at the end of the study (Table 3). The mean total SGRQ score at the first visit in the roxithromycin group was $43.8 \pm 14.9$ points which was similar to the score of $55.6 \pm 18.0$ points in the placebo group $(p-$ value $=1.00)($ Table 4). The SRGQ scores at the 8-week visit are shown in Table 4. The mean differences of the SGRQ scores between the roxithromycin and placebo groups showed no statistically significant differences in any of the domains (symptoms, activity, impact, and overall) despite statistically significant improvement of the SGRQ scores in each group compared with the SGRQ scores at the baseline visit (symptoms, impact, and overall domains) (Figure 2).

The most common bacterial colonizations in the roxithromycin and placebo groups were Pseudomonas aeruginosa and Klebsiella pneumoniae. No bacterial resistant strains were reported at the end of the study. None of the patients who received roxithromycin reported any adverse side effects. However, three patients in the placebo group had minor adverse side effects. One patient experienced abdominal bloating, one patient had dry mouth, and one patient had a worsening cough.

Table 1 Baseline characteristics

\begin{tabular}{lll}
\hline Variables & $\begin{array}{l}\text { Roxithromycin } \\
(\mathbf{n}=12)\end{array}$ & $\begin{array}{l}\text { Placebo } \\
(\mathbf{n}=14)\end{array}$ \\
\hline $\begin{array}{ll}\text { Age, yr (min-max) } \\
\text { Sex, n male/female }\end{array}$ & $55(38-69)$ & 60 (39-78) \\
Smoking status, $\mathrm{n}$ & $8 / 4$ & $6 / 8$ \\
$\quad$ Current & 0 & 2 \\
$\quad$ Exsmoker & 4 & 4 \\
$\quad$ Never & 8 & 8 \\
Etiology of bronchiectasis, $\mathrm{n}$ & \\
$\quad \begin{array}{l}\text { Post-tuberculosis } \\
\quad \text { Post-pneumonia }\end{array}$ & 7 & 8 \\
$\quad$ Unknown & 1 & 2 \\
Concurrent respiratory medication & 4 & 4 \\
$\quad$ Inhaled corticosteroid & 4 & 6 \\
Long acting beta-2-agonist & & \\
$\quad$ Theophylline & 5 & 7 \\
$\quad$ Mucolytic & 7 & 10 \\
Median number of exacerbations & 3 & 3 \\
in prior year & & \\
\hline
\end{tabular}


Table 2 Primary outcome: symptom scores

\begin{tabular}{lllll}
\hline Study group & $\begin{array}{l}\text { First visit symptoms } \\
\text { scores }\end{array}$ & $\begin{array}{l}\text { Second visit symptoms } \\
\text { scores }\end{array}$ & $\begin{array}{l}\text { Mean difference of } \\
\text { symptoms scores }\end{array}$ & $\begin{array}{l}\mathbf{p} \text {-value } \\
\text { Roxithromycin }(\mathrm{n}=12)\end{array}$ \\
Placebo $(\mathrm{n}=14)$ & $5.33 \pm 1.72$ & $*$ & $-1.66 \pm 1.23$ & 0.027 \\
& $5.35 \pm 1.98$ & $\begin{array}{l}3.67 \pm 1.82 \\
* *\end{array}$ & $-0.06 \pm 2.34$ \\
\hline
\end{tabular}

Values are presented as mean \pm S.D., compared mean difference between roxithromycin and placebo.

${ }^{*} p$-value $=0.005,{ }^{* *} p$-value $=0.094$ compared to the value before treatment.

Table 3 Primary outcomes: pulmonary function tests

\begin{tabular}{|c|c|c|c|c|}
\hline Pulmonary function test & First visit & Second visit & Mean difference & $p$-value \\
\hline $\mathrm{FEV}_{1}, \mathrm{~L}$ & & & & 0.870 \\
\hline Roxithromycin & $1.53 \pm 0.62$ & $1.49 \pm 0.61$ & $-0.04 \pm 1.23$ & \\
\hline Placebo & $1.31 \pm 0.44$ & $1.34 \pm 0.47$ & $0.03 \pm 0.27$ & \\
\hline FVC, L & & & & 0.870 \\
\hline Roxithromycin & $2.27 \pm 0.79$ & $2.29 \pm 0.70$ & $0.02 \pm 0.17$ & \\
\hline Placebo & $1.98 \pm 0.55$ & $1.91 \pm 0.70$ & $-0.07 \pm 0.38$ & \\
\hline KCO, percent & & & & 0.710 \\
\hline Roxithromycin & $84.00 \pm 15.62$ & $88.12 \pm 19.28$ & $4.12 \pm 15.47$ & \\
\hline Placebo & $83.90 \pm 30.19$ & $86.54 \pm 28.58$ & $2.64 \pm 14.44$ & \\
\hline
\end{tabular}

Values are presented as mean \pm S.D., compared mean difference between roxithromycin and placebo.

$\mathrm{FEV}{ }_{1}=$ force expiratory volume in 1 second, $\mathrm{FVC}=$ force vital capacity, $\mathrm{KCO}=$ transfer coefficient of the lung for carbon monoxide, $\mathrm{L}=$ liter

Table 4 Secondary outcomes: quality of life measured by St. George's Respiratory Questionnaire

\begin{tabular}{|c|c|c|c|c|}
\hline SGRQ & First visit & Second visit & Mean difference & $p$-value \\
\hline Symptoms & & & & 0.350 \\
\hline Roxithromycin & $58.00 \pm 13.80$ & $39.90 \pm 16.90$ & $-18.10 \pm 22.50$ & \\
\hline Placebo & $68.80 \pm 22.90$ & $57.70 \pm 23.60$ & $-11.10 \pm 19.60$ & \\
\hline Activity & & & & 0.770 \\
\hline Roxithromycin & $45.50 \pm 23.50$ & $36.50 \pm 19.70$ & $-9.00 \pm 20.50$ & \\
\hline Placebo & $58.90 \pm 23.90$ & $50.20 \pm 24.10$ & $-8.70 \pm 27.70$ & \\
\hline Impact & & & & 0.270 \\
\hline Roxithromycin & $38.80 \pm 13.90$ & $28.30 \pm 15.10$ & $-10.50 \pm 12.60$ & \\
\hline Placebo & $49.90 \pm 20.60$ & $41.60 \pm 20.40$ & $-8.30 \pm 18.50$ & \\
\hline Overall & & & & 0.470 \\
\hline Roxithromycin & $43.80 \pm 14.90$ & $32.70 \pm 14.90$ & $-11.10 \pm 11.60$ & \\
\hline Placebo & $55.60 \pm 18.00$ & $46.60 \pm 19.80$ & $-9.00 \pm 16.30$ & \\
\hline
\end{tabular}

$S G R Q=S t$. George's Respiratory Questionnaire 


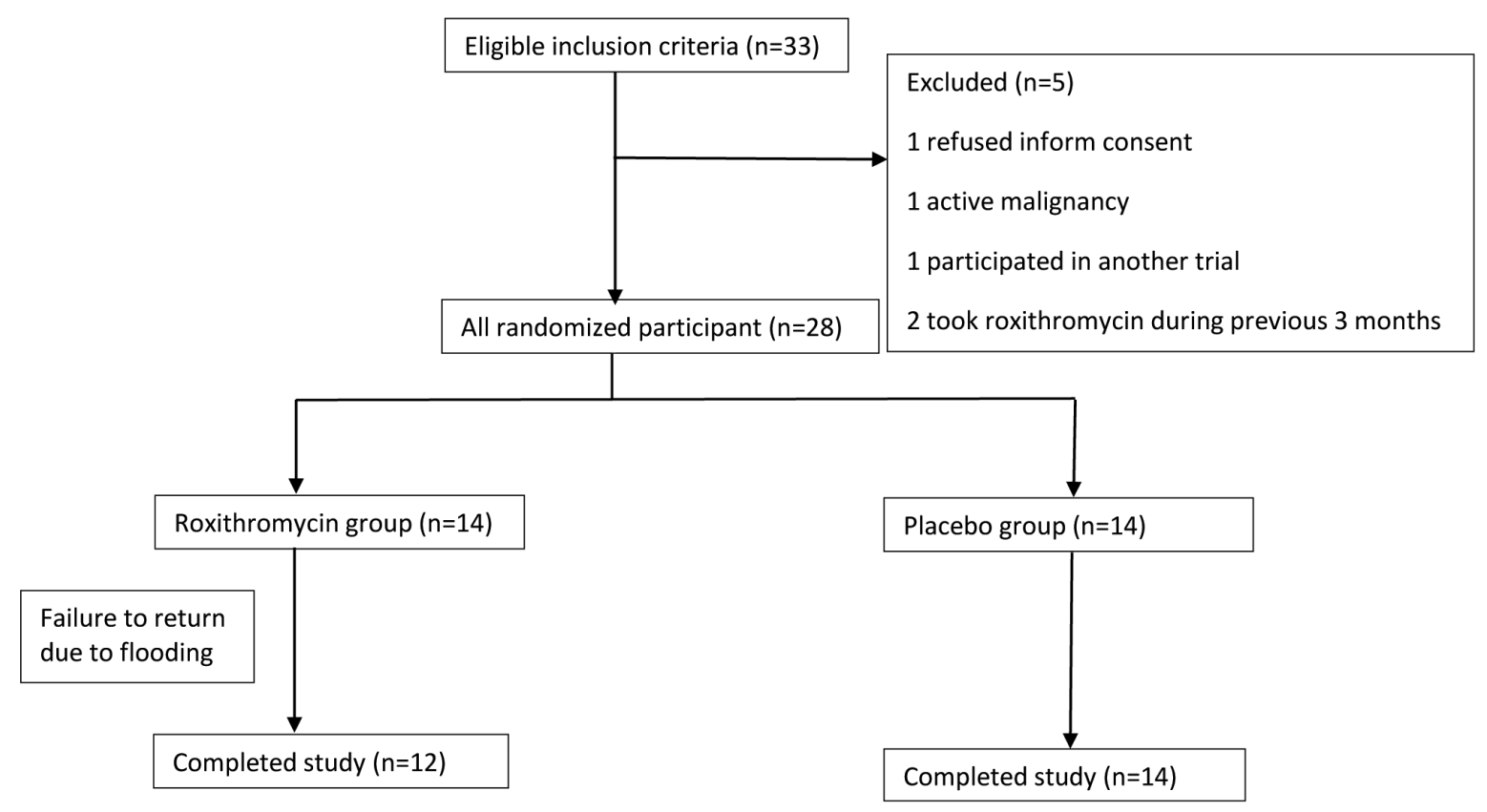

Figure 1 Flow chart of the study

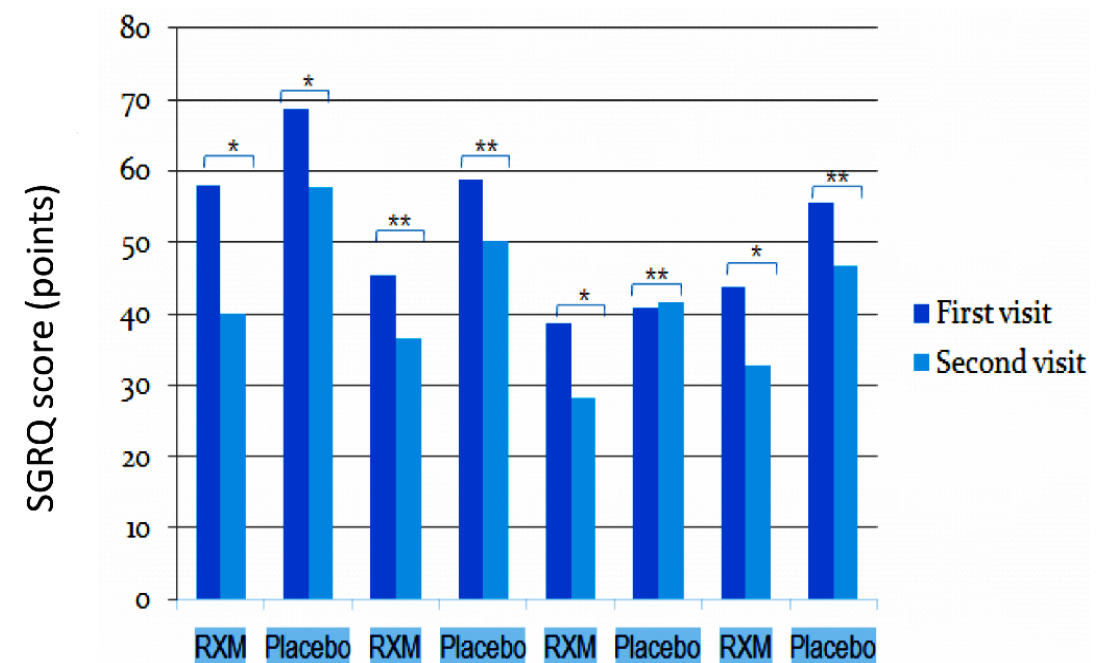

Symptom Activity Impact Overall

SGRQ domain

Values are presented as mean \pm S.D., compared mean difference between roxithromycin and placebo that compared the value before and after treatment

${ }^{*} \mathrm{p}$-value $<0.05,{ }^{* *} \mathrm{p}$-value $>0.05, \mathrm{RXM}=$ roxithromycin, $S \mathrm{GRQ}=\mathrm{St}$. George's Respiratory Questionnaire

Figure 2 Comparisons of St. George's Respiratory Questionnaire between first and second visit after 8 weeks of treatment 


\section{Discussion}

We conducted a randomized double-blind placebocontrolled trial to evaluate the efficacy of roxithromycin as an anti-inflammatory agent on clinical outcomes in adult patients with bronchiectasis. Roxithromycin showed benefits on the clinical outcomes compared with placebo, especially improvements in the symptom scores (e.g. cough, purulent sputum production, wheezing, and dyspnea) as well as quality of life (especially symptoms, impact, and overall domains but not activity domain) despite no beneficial effect on pulmonary function.

The burdens of bronchiectasis patients are daily symptoms and recurrent infectious exacerbations which lead to poor quality of life. The benefits of long-term macrolides as anti-inflammatory drugs in bronchiectasis to reduce infectious exacerbations were established from three large randomized trials using long-term azithromycin ${ }^{7,8}$ and erythromycin. ${ }^{6}$ In our study, roxithromycin significantly improved the symptom scores compared with placebo because long-term roxithromycin suppresses airway inflammation measured by the decrease of sputum inflammatory markers such as interleukin-8 and reduces chronic airway damage by decreased remodeling of dilated bronchial walls in patient with bronchiectasis, ${ }^{10}$ which correlated with improved daily symptoms of bronchiectasis patients.

In our study, roxithromycin 300 mg daily significantly improved the quality of life evaluated by the SGRQ symptoms domain in patients with bronchiectasis. The same improved quality of life was reported from a recent open-label study of low dose roxithromycin $150 \mathrm{mg}$ daily for 6 months which improved in the SGRQ symptoms domain and total scores. ${ }^{10}$ In a comparison of large randomized control trials, only azithromycin $250 \mathrm{mg}$ daily for 12 months had a statistically improved quality of life but not at 6 months. ${ }^{8}$ Also, neither erythromycin at 48 weeks nor azithromycin 500 mg daily at 6 months demonstrated improved quality of life. ${ }^{6,7}$ Our bronchiectasis patients had high baseline $S G R Q$ scores which indicated poor quality of life. Interestingly, both roxithromycin and placebo provided improvement in the SGRQ scores due to the enrollment to this clinical study.

The reason roxithromycin had no benefit on pulmonary functions in this study could be from the small sample size and short duration of follow-up. However, data from a meta-analysis reported that macrolides attenuated a decline in $\mathrm{FEV}_{1}$ of only 0.02 liter which was not clinically relevant. ${ }^{11}$ The benefit on lung function might need longer follow-up time. Furthermore, the natural course of bronchiectasis in patients includes intermittent episodes of exacerbations which results in poorer lung function.

Roxithromycin in our study demonstrated a good safety profile with no significant adverse side effects and no evidence of emerged bacterial resistant strains at the end of treatment. The patients tolerated roxithromycin quite well. Despite an in vitro study that showed similar pharmacokinetic and dynamic properties of roxithromycin compared with azithromycin, roxithromycin had a good safety profile, fewer cardiovascular adverse effects, and a lower price of roxithromycin compared with azithromycin. ${ }^{12,13}$ Roxithromycin is the optimal choice for long-term macrolide therapy in bronchiectasis patients.

This study had some limitations. The sample size was small and the duration of roxithromycin of 8 weeks was short. The data from an in vitro study showed that the anti-inflammatory properties of roxithromycin were found after 7 weeks of treatment. ${ }^{14}$ For that reason, we chose an 8 week treatment duration of the macrolide. Our study had no data on the effect of roxithromycin for exacerbation in bronchiectasis patients. In an open-label design study, roxithromycin $150 \mathrm{mg}$ daily for 6 months reported significantly delayed time to the first exacerbation compared with control patients $(p-$ value $=0.022) .{ }^{10}$ 


\section{Conclusion}

Roxithromycin is significantly beneficial on clinical outcomes. There were improvements in the symptom scores and quality of life but no benefit on pulmonary functions in patient with bronchiectasis. Roxithromycin had a good safety profile and it was well tolerated. Furthermore, there was an absence of emerged resistant bacteria. However, a larger population, longer duration of follow-up, and a multicenter study are required to confirm the effects of roxithromycin compared with other long-term macrolide therapy in bronchiectasis patients.

\section{Acknowledgement}

The authors thanks to all participants in this study.

\section{Conflict of interest}

The authors had no conflict of interest in this study.

\section{References}

1. McShane PJ, Naureckas ET, Tino G, Strek ME. Non-cystic fibrosis bronchiectasis. Am J Respir Crit Care Med 2013;188: 647-56.

2. Barker AF. Bronchiectasis. N Engl J Med 2002;346:1383-93.

3. Cole P. Inflammation: a two-edged sword-the model of bronchiectasis. Eur J Respir Dis Suppl 1986;147:6-15.

4. Moulton BC, Barker AF. Pathogenesis of bronchiectasis. Clin Chest Med 2012;33:211-7.

5. Crosbie PAJ, Woodhead MA. Long-term macrolide therapy in chronic inflammatory airway disease. Eur Respir J 2009;33: 171-81.

6. Serisier DJ, Martin ML, McGuckin MA, Lourie R, Chen AC, Brain
B, et al. Effect of long-term, low-dose erythromycin on pulmonary exacerbations among patients with non-cystic fibrosis bronchiectasis. The BLESS randomized controlled trial. JAMA 2013;309:1260-7.

7. Wong C, Jayaram L, Karalus N, Eaton T, Tong C, Hockey H, et al. Azithromycin for prevention of exacerbations in noncystic fibrosis bronchiectasis (EMBRACE): a randomised, double-blind, placebo-controlled trial. Lancet 2012;380:660-7.

8. Altenburg J, de Graaff CS, Stienstra Y, Sloos JH, van Haren EH, Koppers RJ, et al. Effect of azithromycin maintenance treatment on infectious exacerbations among patients with non-cystic fibrosis bronchiectasis. The BAT randomized controlled trial. JAMA 2013;309:1251-9.

9. Nakamura H, Fujushima S, Inoue T, Ohkubo $Y$, Soejima K, Waki $Y$, et al. Clinical and immunoregulatory effects of roxithromycin therapy for chronic respiratory tract infection. Eur Respir J 1999;13:1371-9.

10. Liu J, Zhong X, He Z, Wei L, Zheng X, Zhang J, et al. Effect of low-dose, long-term roxithromycin on airway inflammation and remodeling of stable noncystic fibrosis bronchiectasis. Mediators Inflamm 2014;2014:708608.

11. Wu Q, Shen W, Cheng H, Zhou X. Long-term macrolides for non-cystic fibrosis bronchiectasis: a systematic review and meta-analysis. Respirology 2014;19:321-9.

12. Young RA, Gonzalez JP, Sorkin EM. Roxithromycin: a review of its antibacterial activity, pharmacokinetic properties and clinical efficacy. Drugs 1989;37:8-41.

13. Surendra K, Puri B, Howard BL. Roxithromycin: a pharmacokinetic review of a macrolide. J Antimicrob Chemother 1987; 20:89-100.

14. Suzaki H, Asano K, Ohki S, Kanai K, Mizutani T, Hisamitsu T, et al. Suppressive activity of a macrolide antibiotic, roxithromycin, on pro-inflammatory cytokine production in vitro and in vivo. Mediators Inflamm 1999;8:199-204. 